\title{
Intersections of quotient rings of an integral domain
}

\author{
By \\ Robert GilmeR ${ }^{1)}$, and William J. HeinZeR \\ (Communicated by Prof. Nagata, Aug. 10, 1967)
}

Let $D$ be an integral domain with identity having quotient field $K$. A domain between $D$ and $K$ is called an overring of $D$, and a valuation overring of $D$ is an overring of $D$ which is also a valuation ring. Davis [2], Gilmer and Ohm [6], Goldman [8], and Pendleton [15] have recently considered domains $D$ with the $Q R$-property: Each overring of $D$ is a quotient ring of $D$. A domain with the $Q R$-property is necessarily Prüfer [2; pp. 197-8], $[6 ;$ p. 99], and a Noetherian domain has the $Q R$-property if and only if it is a Dedekind domain with torsion class group [2; p. 200], [6; p. 100], [8; p. 114].

Davis in [2] and Gilmer in [4] have considered a related property on a domain $D$, which we shall refer to here as the $Q Q R$ property: Each overring of $D$ is an intersection of quotient rings of $D$. Since any quotient ring of $D$ is an intersection of localizations of $D$ (that is, quotient rings of $D$ taken with respect to the complement of prime ideals of $D$ ), the $Q Q R$-property for $D$ is equivalent to the condition that each overring of $D$ is an intersection of localizations of $D$. It is well-known that any Dedekind domain has the $Q Q R$-property ; more generally, any Prüfer domain has the $Q Q R$-property [2; p. 197]. Davis in [2; p. 200] raises the question of the validity of the converse; he proves that if each prime ideal of $D$ is of finite rank and if $D$ has the $Q Q R$ -

1) The first author was supported during the writing of this paper by National Science Foundation Grant GP-4127. 
property, then $D$ is Prüfer. In Section 1 we consider more closely the relation between the concepts " $D$ is Prüfer" and " $D$ has the $Q Q R$-property". We are able to generalize the positive result of Davis just cited (Theorem 1.4) and to add a second positive result (Corollary 1.7), but we give an example later in Section 4 showing that a domain with the $Q Q R$-property need not be Prüfer. Theorem 1.9 shows that the domain $D$ has the $Q Q R$-property if and only if $D_{M}$ has the $Q Q R$-property for each maximal ideal $M$ of $D$. Theorem 1.10 states that if $J$ is a quasi-local domain with the $Q Q R$-property which is not integrally closed, then the integral closure $\bar{J}$ of $J$ is the unique minimal overring of $J$ in the sense that each proper overring of $J$ contains $\bar{J}$. Hence Section 2 is devoted to a consideration of domains which admit a unique minimal overring, and Theorem 3.3 of Section 3 gives a characterization of quasi-local domains with the $Q Q R$-property.

All domains considered in this paper are assumed to contain an identity. The terminology is that of Zariski-Samuel [17] [18].

1. Prüfer domains and the $Q Q R$-property. We establish in this section some consequences of the $Q Q R$-property in a domain $D$. Theorem 1.4 and Corollary 1.7 relate the $Q Q R$-property to the property of being Prüfer. We shall have frequent occasion to use the following result from [4], which we quote directly:

Suppose $D^{\prime}$ is an overring of the Prïfer domain $D$, and let $\Omega$ be the set of prime ideals $P$ of $D$ such that $P D^{\prime} \subset D^{\prime}$. Then

(i) If $M$ is a maximal ideal of $D^{\prime}$ and if $P=M \cap D$, then $D_{P}=D^{\prime}{ }_{M}$ and $M=P D_{P} \cap D^{\prime}$. Therefore $D^{\prime}$ is Prüfer.

(ii) For $P$ a proper prime ideal of $D, P \in \Omega$ if and only if $D_{P} \supseteq D^{\prime}$. Further, $D^{\prime}=\cap_{P \in \Omega} D_{P}$.

(iii) If $A^{\prime}$ is an ideal of $D^{\prime}$ and $A=A^{\prime} \cap D$, then $A^{\prime}=A D^{\prime}$.

(iv) $\left\{P D^{\prime}\right\}_{P \in \Omega}$ is the set of proper prime ideals of $D^{\prime}$.

Also, we shall make use of the following fact concerning localizations.

If $D^{\prime}$ is an overring of the domain $D$ and $P$ is a prime ideal of $D$ such that $D^{\prime} \subseteq D_{P}$, then $D_{P^{\prime}}^{\prime}=D_{P}$ where $P^{\prime}=D^{\prime} \cap P D_{P}$.

1.1 PROPOSITION. Let $D$ be an integral domain and let $D^{\prime}$ be 
an overring of $D$. If $D^{\prime}$ is an intersection of quotient rings of $D$ then $D^{\prime}$ is an intersection of localizations of $D$.

Proposition 1.1 is immediate from the easily-proved fact that a quotient ring of $D$ is an intersection of localizations of $D$. (For example, see [16].)

1.2 COROLLARY. The following statements concerning the integral domain $D$ are equivalent.

(1) D has the QQR-property.

(2) Each overring of $D$ is an intersection of localizations of $D$.

(3) Each overring of $D$ which is quasi-local is an intersection of localizations of $D$.

Proof. In view of Proposition 1.1 we need only show that (3) implies (1). Thus suppose $D^{\prime}$ is an overring of $D$ and $\left\{M_{\alpha}\right\}$ is the collection of maximal ideals of $D^{\prime}$. By hypothesis, $D^{\prime}{ }_{M_{\alpha}}$ is an intersection of localizations of $D$. Since $D^{\prime}=\bigcap_{\alpha} D_{{ }_{M_{\alpha}}}$, (1) then follows.

In considering questions concerning overrings of the domain $D$ we find that an important role is played by the valuation overrings of $D$-that is, valuation rings lying between $D$ and $K$. Our first results therefore deal with the condition that every valuation overring of $D$ is an intersection of quotient rings of $D$.

1.3 LEMMA. Let $V$ be a valuation overring of $D$ which is an intersection of localizations of $D: V=\prod_{\alpha} D_{P_{\alpha}}$. Then each $D_{P_{\alpha}}$ is a valuation ring, the set $\left\{P_{\alpha}\right\}$ is linearly ordered under inclusion, and $M=\bigcup_{\alpha} P_{\infty}$ is the center of $V$ on $D . \quad$ Also, $D_{M} \subseteq V$ and equality holds if and only if $D_{M}$ is a valuation ring. If $A$ is a nonmaximal proper ideal of $V$, then $A \subset P_{\alpha} D_{P_{\alpha}}$ for some $\alpha$.

Proof. Since each $D_{P_{\alpha}}$ contains $V$, each $D_{P_{\alpha}}$ is a valuation ring and $\left\{P_{\alpha} D_{P_{\alpha}}\right\}$ is chained under inclusion. Because $P_{\alpha}=$ $P_{\alpha} D_{P_{\alpha}} \cap D$ for each $\alpha,\left\{P_{\alpha}\right\}$ is also chained under inclusion. Therefore $M=\bigcup_{\alpha} P_{\alpha}$ is prime in $D$. We next observe that $\bigcup_{\alpha} P_{a} D_{P_{\alpha}}$ is the maximal ideal $M^{\prime}$ of $V$. That $\bigcup_{\alpha} P_{\alpha} D_{P_{a}} \subseteq M^{\prime}$ is clear. And if $x$ is a nonunit of $V, 1 / x \notin V=\bigcap_{a} D_{P_{a}}$ implies $1 / x \notin D_{P_{a}}$ for some 
$\alpha$. Hence $M^{\prime} \subseteq \bigcup_{\alpha} P_{\alpha} D_{P_{\alpha}}$. Therefore the center of $V$ on $D$ is $M^{\prime} \cap D=\left(\cup P_{\alpha} D_{P_{\alpha}}\right) \cap D=\bigcup_{\alpha}\left(P_{\alpha} D_{P_{\alpha}} \cap D\right)=\bigcup_{\alpha} P_{\alpha}=M$. It follows that $D_{M} \subseteq V$. If $D_{M}$ is a valuation ring, then $D_{M}=V$, for $M \subseteq M^{\prime}$ implies that $M D_{M} \subseteq M V \subseteq M^{\prime} ; M D_{M}$ the maximal ideal of $D_{M}$. Finally, if $A$ is an ideal of $V$ such that $A \subset M^{\prime}$, then $A \nsubseteq \bigcup_{\alpha} P_{\alpha} D_{P_{\alpha}}$ so $A \nsubseteq P_{\alpha} D_{P_{\alpha}}$ for some $\alpha$. Hence $A \subset P_{\alpha} D_{P_{\alpha}}$.

1.4 THEOREM. If each valuation overring of $D$ is an intersection of quotient rings of $D$, and if $D$ satisfies the ascending chain condition (a.c.c.) for prime ideals then $D$ is a Prïfer domain.

Proof. Let $M$ be a maximal ideal of $D$. There is a valuation overring of $D$ having center $M$ on $D$. By Lemma 1.3, there is a chain $\left\{P_{\alpha}\right\}$ of prime ideal of $D$ such that $\bigcup_{\alpha} P_{\alpha}=M$ and each $D_{P_{\alpha}}$ is a valuation ring. Since $D$ satisfies a.c.c. on prime ideals, some $P_{\alpha}$ is equal to $M$, and $D_{M}$ is a valuation ring. It follows that $D$ is a Prüfer domain.

1.5 THEOREM. If each valuation overring of $D$ is an intersection of quotient rings of $D$, then for $P$ a nonmaximal prime of $D, D_{P}$ is a valuation ring.

Proof. Let $M$ be a maximal ideal of $D$ such that $P \subset M$ and let $V$ be a valuation overring of $D$ having prime ideals $P^{\prime}$ and $M^{\prime}$ such that $P^{\prime} \cap D=P, M^{\prime} \cap D=M$, and $M^{\prime}$ is the maximal ideal of $V$. [13, p. 37]. By Lemma 1.3 there is a set $\left\{P_{\alpha}\right\}$ of prime ideals of $D$ such that $V=\bigcap_{\alpha} D_{P_{\alpha}}$, and $P^{\prime} \subset P_{\alpha} D_{P_{\alpha}}$ for some $\alpha$. Therefore, $P=P^{\prime} \cap D \subseteq P_{\alpha} D_{P_{\alpha}} \cap D=P_{\alpha}$. It follows that $D_{P_{\alpha}} \subseteq D_{P}$, and $D_{P}$ is a valuation ring as we wished to show.

1.6 THEOREM. If each valuation overring of $D$ is an intersection of quotient rings of $D$, then $\bar{D}$, the integral closure of $D$, is a Prüfer domain.

Proof. If $\bar{D}$ is not a Prüfer domain, then there is a maximal ideal $\bar{M}$ of $\bar{D}$ such that $\bar{D}_{\bar{M}}$ is not a valuation ring. It follows (see, for example, $[18$, p. 21]) that there is a valuation overring $V$ of $\bar{D}$ such that $V$ has prime ideals $P^{\prime} \subset M^{\prime}$ with $P^{\prime} \cap \bar{D}=M^{\prime} \cap \bar{D}$ 
$=\bar{M}$. Hence if $\bar{M} \cap D=M$, then $P^{\prime} \cap D=M$ also. By hypothesis $V$ is an intersection of quotient rings of $D$. Thus, there is a set $\left\{P_{\alpha}\right\}$ of prime ideals of $D$ such that $V=\bigcap_{\alpha} D_{P_{\alpha}}$, and $P^{\prime} \subset P_{\alpha} D_{P_{\alpha}}$ for some $\alpha$. This means that $M \subseteq P^{\prime} \cap D \subseteq P_{\alpha} D_{P_{\alpha}} \cap D=P_{\alpha}$; hence $M=P_{\alpha}$ and $D_{M}=D_{P_{\alpha}}$ is a valuation ring. But $\bar{M} \cap D=M$ implies that $D_{M} \subseteq \bar{D}_{\bar{M}}$, and by assumption $\bar{D}_{\bar{M}}$ is not a valuation ring. This contradiction establishes Theorem 1.6.

1.7 COROLLARY ${ }^{2}$. If $D$ has the $Q Q R$-property, then $\bar{D}$, the integral closure of $D$, is Prïfer. Therefore if $D$ is an integrally closed domain, $D$ has the QQR-property if and only if $D$ is a Prïfer domain.

If $J$ is a domain lying between a domain $D$ and a quotient ring $D_{N}$ of $D$, then $D_{N}=J_{N}$. Therefore we have

1.8 PROPOSITION. If $D$ is a domain with the $Q Q R$-property and if $D^{\prime}$ is an overring of $D$, then $D^{\prime}$ has the QQR-property.

Our next result reduces the problem of characterizing domains with the $Q Q R$-property to the study of quasi-local domains.

1.9 THEOREM. The domain $D$ has the $Q Q R$-property if and only if $D_{M_{\infty}}$ has the $Q Q R$-property for each maximal ideal $M_{a}$ of $D$.

Proof. If $D$ has the $Q Q R$-property, then by Proposition 1.8 each $D_{M_{a}}$ has the $Q Q R$-property. We assume, conversely, that each $D_{M_{a}}$ has the $Q Q R$-property. By Proposition 1.2, $D$ will have the $Q Q R$-property if each quasi-local overring of $D$ is an intersection of localizations of $D$. Hence, let $D^{\prime}$ be a quasi-local overring of $D$ with maximal ideal $M^{\prime}$. If $P=M^{\prime} \cap D$ and if $M_{\alpha}$ is a maximal ideal of $D$ containing $P$, then $D_{M_{\alpha}} \subseteq D_{P} \subseteq D^{\prime}$. Since $D_{M_{\alpha}}$ has the $Q Q R$-property, $D^{\prime}$ is an intersection of localizations of $D_{M_{\alpha}}$. But each localization of $D_{M_{\alpha}}$ is a localization of $D$, hence $D^{\prime}$ is an intersection of localizations of $D$ as we wished to show.

TERMINOLOGY. We will say that the overring $D_{1}$ of the

2) Corollary 1.7 can also be obtained as a special case of Corollary 1 of [2]. A domain $D$ such that each valuation overring of $D$ is an intersection of quotient rings of $D$ need not have the $Q Q R$-property. Example 4.2 illustrates this fact. 
domain $D$ is the unique minimal overring of $D$ if $D \subset D_{1}$ and if for any overring $D_{2}$ of $D$ not equal to $D$ we have $D_{1} \subseteq D_{2}$.

1.10 THEOREM. Let $D$ be a quasi-local domain with maximal ideal $M$. If $D$ has the QQR-property and is not a valuation ring, then $\bar{D}$, the integral closure of $D$, is the unique minimal overring of $D$.

Proof. Since $D$ is quasi-local and is not a valuation ring, $D$ is not Prüfer, so that $D \subset \bar{D}$. If $D^{\prime}$ is an overring of $D$, there is a set $\left\{P_{\alpha}\right\}$ of prime ideals of $D$ such that $D^{\prime}=\bigcap_{\alpha} D_{P_{\infty}}$, and if $D \neq D^{\prime}$, then $M \notin\left\{P_{\alpha}\right\}$ since $D_{M}=D$. Therefore each $P_{\alpha}$ is nonmaximal, and Theorem 1.5 shows that each $D_{P_{\alpha}}$ is then a valuation ring. Therefore $D^{\prime}=\bigcap_{\alpha} D_{P_{\alpha}}$ is an integrally closed domain containing $D$. Hence $\bar{D} \subseteq D^{\prime}$, and we conclude that $\bar{D}$ is the unique minimal overring of $D$.

2. Unique minimal overrings. Theorem 1.10 leads us to an investigation of domains which possess a unique minimal overring. From the fact that a domain is the intersection of the localizations taken with respect to its set of maximal ideals, it follows that a domain which has a unique minimal overring is quasi-local. Also, since an integrally closed domain is an intersection of valuation rings, if $D$ is integrally closed and has a unique minimal overring, then $D$ is a valuation ring. Our primary interest lies in the case where $D$ is quasi-local and $\bar{D}$, the integral closure of $D$, is the unique minimal overring of $D$.

We consider first the situation when $D$ and $D_{1}$ are domains with $D \subset D_{1}$ such that there are no domains properly between $D$ and $D_{1}$.

2.1 LEMMA. If $A$ and $B$ are distinct ideals of $D_{1}$ such that $A \cap D=B \cap D$, then $A \cap B=A \cap D$. Therefore, $A \cap B$ is an ideal of $D$.

Proof. We have $A \cap D=(A \cap D) \cap(B \cap D) \subseteq A \cap B$. If $A \cap D \subset$ $A \cap B$, then there is an $x \in(A \cap B)-D$, and since there are no domains properly between $D$ and $D_{1}$, we have $D_{1}=D[x]$. If $y \in A$, then $y=d_{0}+d_{1} x+\cdots+d_{n} x^{n}$ where $d_{0}, d_{1}, \cdots, d_{n} \in D$. Hence $d_{0}=y-d_{1} x-\cdots-d_{n} x^{n} \in A \cap D=B \cap D$, and $y=d_{0}+d_{1} x+\cdots+d_{n} x^{n} \in B$. 
It follows that $A \subseteq B$; similarly $B \subseteq A$, so that $A=B$. Consequently, if $A$ and $B$ are distinct ideals of $D_{1}$ such that $A \cap D=B \cap D$, then $A \cap B=A \cap D$.

2.2 COROLLARY. If $P$ is a prime ideal of $D$, then there are at most two prime ideals of $D_{1}$ lying over $P$. In particular, if $D$ is quasi-local and $D_{1}$ is integral over $D, D_{1}$ has at most two maximal ideals.

2.3 LEMMA. Assume that $D$ is quasi-local with maximal ideal $M$. If $D_{1}$ is integral over $D$, then $M$ is the conductor of $D$ in $D_{1}$.

Proof. Let $y \in M$ and assume that $y D_{1} \mp D$. Then there is an $x \in y D_{1}-D$; say $y \xi=x$. Since $x \notin D$, since there are no domains properly between $D$ and $D_{1}$, and since $D_{1}$ is integral over $D$, we have $D_{1}=D+D x+\cdots+D x^{n}$ for some positive integer $n$. We choose $n$ minimal with this property. There are $d_{0}, \cdots, d_{n} \in D$ such that $\xi^{n}=d_{0}+d_{1} x+\cdots+d_{n} x^{n}$. Hence $x^{n}=y^{n} \xi^{n}=y^{n} d_{0}+\cdots+y^{n} d_{n} x^{n}$ and $x^{n}\left(1-y^{n} d_{n}\right)=y^{n} d_{0}+\cdots+y^{n} d_{n-1} x^{n-1}$. Since $D$ is quasi-local and $y \in M$, $1-y^{n} d_{n}$ is a unit of $D$ and $x^{n} \in D+D x+\cdots+D x^{n-1}$. It follows that $D_{1}=D+D x+\cdots+D x^{n-1}$, which contradicts our choice of $n$. We conclude that $M$ is the conductor of $D$ in $D_{1}$.

2.4 THEOREM. Let $D$ be a quasi-local domain with maximal ideal $M$ and integral closure $\bar{D}$ such that $D \subset \bar{D}$. If $\bar{D}$ is Prüfer and if there are no domains properly between $D$ and $\bar{D}$, then $\bar{D}$ is the unique minimal overring of $D$.

Proof. By Lemma 2.3, $M$ is the conductor of $D$ in $\bar{D}$ and by Corollary 2.2, $\bar{D}$ has at most two maximal ideals. For $x \in K-D$ we show that $\bar{D} \subseteq D[x]$. If $x \in \bar{D}-D$, then $\bar{D}=D[x]$ by hypothesis. If $\bar{D}$ is quasi-local with maximal ideal $\bar{M}$, then since $\bar{D}$ is integral over $D, \bar{M}$ is the only prime of $\bar{D}$ lying over $M$. Hence $M=M \bar{D}$ has radical $\bar{M}$ in $\bar{D}$. For $x \notin \bar{D}$, we have $1 / x \in \bar{M}$, since $\bar{D}$ is a valuation ring, so that $(1 / x)^{n} \in M$ for some positive integer $n$. Thus for $\xi \in \bar{D}$, we have $\xi=\xi\left(1 / x^{n}\right) x^{n} \in D[x]$ and $\bar{D} \subseteq D[x]$.

The remaining case is when $\bar{D}$ has two maximal ideals-say $M_{1}$ and $M_{2}$. By Lemma 2.1, $M=M_{1} \cap M_{2}$ and since $\bar{D}$ is Prüfer 
$\bar{D}_{M_{1}}$ and $\bar{D}_{M_{2}}$ are valuation rings. For $x \notin \bar{D}$, we have $x \notin \bar{D}_{M_{1}}$ or $x \notin \bar{D}_{M_{2}}$. Assume $x \notin \bar{D}_{M_{1}}$; then $1 / x \in M_{1} \bar{D}_{\hat{M}_{\hat{1}}}$. Hence $1 / x=u / v$ where $u \in M_{1}$ and $v \in \bar{D}-M_{1}$. If $y \in M_{2}-M_{1}$, then $1 / x=u y / v y$ and $v y \in \bar{D}-M_{1}$. Thus $x u y=v y \in \bar{D}-D$. But $u y \in M_{1} \cap M_{2} \subset D$. Hence $\bar{D}=D[v y] \subseteq D[x]$. We conclude that $\bar{D}$ is the unique minimal overring of $D$ and Theorem 2.4 is established.

We remark that without the condition that $\bar{D}$ is a Prüfer domain the conclusion of Theorem 2.4 need not follow. For example if $F=R(\sqrt{2})(x)$, where $R$ is the field of rational numbers and $x$ is transcendental over $R$ then the power series ring $F[[y]]$ is a rank one valuation ring of the form $F+M$ where $M$ is the maximal ideal of $F[[y]] . \quad D=R+M$ is a quasi-local domain with integral closure $\bar{D}=R(\sqrt{2})+M$. There are no domains properly between $D$ and $\bar{D}$; however $\bar{D}$ is not a unique minimal overring of $D$ because $\bar{D} \nsubseteq D[x]$.

We consider now the question of what domains admit a unique minimal overring. As we have already mentioned, if $D$ is an integrally closed domain which has a unique minimal overring, then $D$ is a valuation ring. If $D$ is not integrally closed and has a unique minimal overring $D_{1}$, then $D \subset D_{1} \subseteq \bar{D}$ where $\bar{D}$ is the integral closure of $D$. We show by example in Section 4 that the unique minimal overring $D_{1}$ of $D$ need not be quasi-local. However, Corollary 2.2 shows that $D_{1}$ has at most two maximal ideals. We consider this case in Proposition 2.5.

2.5 PROPOSITION. If $D_{1}$, the unique minimal overring of $D$, has two maximal ideals, then $\bar{D}$, the integral closure of $D$, is an intersection of two valuation rings $V_{1}$ and $V_{2}$. If $N_{i}$ is the maximal ideal of $V_{i}$, then $N_{1} \cap N_{2}=M$, the maximal ideal of $D$.

Proof. Let $M_{1}$ and $M_{2}$ be the maximal ideals of $D_{1}$. If $V_{1}$ and $V_{2}$ are valuation overrings of $D$ such that $V_{i}$ has maximal ideal $N_{i}$, where $N_{i} \cap D_{1}=M_{i}$, and if $x \in\left(N_{1} \cap N_{2}\right)-M$, we have $D_{1} \subseteq D[x]$. Hence if $\xi \in D_{1}, \quad \xi=d_{0}+d_{1} x+\cdots+d_{n} x^{n}$, where $d_{0}$, $\cdots, d_{n} \in D$. Also $\xi-d_{0} \in D_{1}$ and $d_{1} x+\cdots+d_{n} x^{n} \in N_{1} \cap N_{2}$; thus $\xi-d_{0} \in N_{1} \cap N_{2} \cap D_{1}=M_{1} \cap M_{2}$. By Lemma 2.1, $M_{1} \cap M_{2}=M \subseteq D$. Therefore the assumption that $x \in\left(N_{1} \cap N_{2}\right)-M$ implies that 
$\xi-d_{0} \in D$ and hence $\xi \in D$. This contradicts the fact that $D \subset D_{1}$. We conclude that $N_{1} \cap N_{2}=M$. If a valuation ring $V_{3}$ is an overring of $D$ and if $N_{3}$, the maximal ideal of $V_{3}$, is such that $N_{3} \cap D=M$, then $D_{1} \subseteq V_{3}$ and $N_{3} \cap D_{1}$ is either $M_{1}$ or $M_{2}$-say $N_{3} \cap D_{1}=M_{1}$. By the argument just given, $N_{3} \cap N_{2}=M$. We show that this implies that $V_{1}=V_{3} . \quad T=V_{1} \cap V_{2} \cap V_{3}$ is a domain such that $T_{N_{i} \cap T}=V_{i}$ for each $i\left[1\right.$, p. 132]. It follows that $\left(N_{3} \cap T\right) \cap\left(N_{2} \cap T\right)=\left(N_{3} \cap N_{2}\right) \cap T$ $=M \cap T \subseteq N_{1} \cap T$. Because there are no containment relations between $V_{1}=T_{N_{1} \cap T}$ and $V_{2}=T_{N_{2} \cap T}$, it follows that $N_{2} \cap T \nsubseteq N_{1} \cap T$. Hence $N_{3} \cap T \subseteq N_{1} \cap T$, and because of the symmetry of our argument, $N_{3} \cap T=N_{1} \cap T$ so that $V_{3}=T_{N_{3} \cap T}=T_{N_{1} \cap T}=V_{1}$. Therefore $V_{1}$ and $V_{2}$ are the only valuation rings which are overrings of $D$ and which have center $M$ on $D$. It follows that $\bar{D}=V_{1} \cap V_{2},[18$, p. 17], and the proof of Proposition 2.5 is complete.

If $D$ is not integrally closed and if $D_{1}$, the unique minimal overring of $D$, is quasi-local with maximal ideal $M_{1}$ then we may have $M_{1}=M$, the maximal ideal of $D$, or $M \subset M_{1}$. When $M \subset M_{1}$, Lemma 2.3 shows that $M$ is an ideal of $D_{1}$ and by Lemma 2.1 there are no ideals properly between $M$ and $M_{1}$. It then follow that $M_{1}^{2} \subseteq M[17$, p. 237]. We have not been able, in the case where $M \subset M_{1}$ and $D_{1}$ is quasi-local, to determine whether the integral closure of $D$ is Prüfer. But if $M_{1}=M$ we show that $\bar{D}$ is a valuation ring.

2.6 PROPOSITION. Let $D_{1}$ be the unique minimal overring of $D$, and assume that $D_{1}$ is quasi-local. If $M$, the maximal ideal of $D$, is also the maximal ideal of $D_{1}$, then $\bar{D}$, the integral closure of $D$, is a valuation ring with maximal ideal $M$.

Proof. Let $V$ be a valuation ring which is an overring of $D$ such that $V$ has center $M$ on $D$. If $N$ is the maximal ideal of $V$, then for $x \in N$ either $x \in M$ or $D_{1} \subseteq D[x]$. But if $D_{1} \subseteq D[x]$, then for $\xi \in D_{1}$ we have $\xi=d_{0}+d_{1} x+\cdots+d_{n} x^{n}$ where $d_{0}, \cdots, d_{n} \in D$. Hence $\xi-d_{0}=d_{1} x+\cdots+d_{n} x^{n} \in N \cap D_{1}=M \subset D$, which means that $\xi \in D$. This contradicts the fact that $D_{1} \nsubseteq D$. Therefore $V$ has maximal ideal $M$ and we conclude that $V=\bar{D}$, the integral closure of $D$ [18, p. 17]. 
We remark that under the hypothesis of Proposition 2.6, $D_{1}$ may be properly contained in $\bar{D}$. In fact, $\bar{D}$ need not be a finite ring extension of $D_{1}$.

We summarize the results of this section as they apply to domains with the $Q Q R$-property.

2.7 THEOREM. Let $D$ be a quasi-local domain with maximal ideal $M$ having the QQR-property. We suppose that $D \subset \bar{D} ; \bar{D}$ the integral closure of $D$. Then $\bar{D}$ is Prüfer with at most two maximal ideals, $\bar{D}$ is the unique minimal overring of $D$, and $M$ is the conductor of $D$ in $\bar{D}$.

Proof. By Theorem 1.10, $\bar{D}$ is the unique minimal overring of $D$. By Corollary $1.7, \bar{D}$ is Prüfer. Corollary 2.2 shows that $\bar{D}$ has at most two maximal ideals, and Lemma 2.3 states that $M$ is the conductor of $D$ in $\bar{D}$.

3. A characterization of quasi-local domains with the $Q Q R$ property. In this section we denote by $D$ a quasi-local domain with maximal ideal $M$ and by $\bar{D}$ the integral closure of $D$.

TERMINOLOGY. We say that a prime ideal $P$ of an integral domain is unbranched if $P$ is the only $P$-primary ideal. Otherwise we say that $P$ is branched $[3$, p. 252]. We remark that a prime ideal $P$ of a Prufer domain is branched if and only if $P$ properly contains the union of the chain of primes properly contained in $P[3]$.

3.1 LEMMA. If $\bar{D}$ is a Prïfer domain with $\left\{M_{\alpha}\right\}$ the set of maximal ideals of $\bar{D}$ and if $M$ is unbranched, then each $M_{\alpha}$ is unbranched.

Proof. If some $M_{\alpha}$ is branched and if for that fixed $\alpha$ we denote by $\left\{Q_{\beta}\right\}$ the set of $M_{\alpha}$-primary ideals, then we have $\bigcap_{\beta} Q_{\beta}=P_{\alpha}$, a prime ideal of $\bar{D}$ properly contained in $M_{\alpha}{ }^{3}$ ) Since $\bar{D}$ is integral over $D, P_{\infty} \cap D=P$ is a prime ideal of $D$ properly

3) Note that this is the only place where the hypothesis that $\bar{D}$ is Prüfer is used; see [14]. 
contained in $M$. Hence there is a $\beta$ such that $Q_{\beta} \cap D \subset M$, and $Q_{\beta} \cap D$ is $M$-primary. Therefore $M$ is branched and Lemma 3.1 is proved.

3.2 LEMMA. Let $\left\{M_{\beta}\right\}$ be the set of maximal ideals of $\bar{D}$. If $M$ is the conductor of $D$ in $\bar{D}$ and if each $M_{\beta}$ is unbranched, then $M$ is unbranched.

Proof. If $Q$ is an $M$-primary ideal of $D$, then since $M$ is an ideal of $\bar{D}, Q M$ is an ideal of $\bar{D}$. Therefore, $Q M=\bigcap_{\beta} Q M \bar{D}_{M_{\beta}}$. We have $Q M \subseteq Q \subseteq M$, and $Q M$ and $M$ have the same radical in $\bar{D}$. Thus, $\left\{M_{B}\right\}$ is the set of minimal primes of $Q M$ in $\bar{D}$ since $\bar{D}$ is integral over $D$. For any $\beta, Q M \bar{D}_{M_{\beta}}$ is primary for $M_{\beta} \bar{D}_{M_{\beta}}$, and hence, $Q M \bar{D}_{M_{\beta}}=M_{\beta} \bar{D}_{M_{\beta}}$ since $M_{\beta}$ is unbranched. It follows that $M \subseteq \bigcap_{\beta} M_{\beta} \bar{D}_{M_{\beta}}=Q M \subseteq Q$ so that $Q=M$ and $M$ is unbranched as we wished to show.

We can give now a characterization of quasi-local domains with the $Q Q R$-property.

3.3 THEOREM. If $D$ is not a valuation ring, the following are equivalent:

(a) D has the QQR-property.

(b) $\bar{D}$ is the unique minimal overring of $D$ and the maximal ideals of $\bar{D}$ are unbranched.

(c) There are no domains properly between $D$ and $\bar{D}, \bar{D}$ is a Prüfer domain, and $M$ is unbranched.

Proof. (a) $\rightarrow$ (b) : By Theorem 1.10, $\bar{D}$ is the unique minimal overring of $D$, and by Corollary $1.7, \bar{D}$ is a Prüfer domain. Hence if $M_{\alpha}$ is a maximal ideal of $\bar{D}, \bar{D}_{M_{\alpha}}$ is a valuation ring. Since $M_{\infty} \cap D=M$, we see that $\bar{D}_{M_{\infty}}$ is an overring of $D$ which is not a quotient ring of $D$. Because $D$ has the $Q Q R$-property, there is a set $\left\{P_{\beta}\right\}$ of prime ideals of $D$ such that $\bar{D}_{M_{\infty}}=\bigcap_{\beta} D_{P_{\beta}}$. Also $D_{P_{\beta}}=\bar{D}_{\bar{P}_{\beta}}$ where $\bar{P}_{\beta}=P_{\beta} D_{P_{\beta}} \cap \bar{D}$. Hence by Lemma 1.3, $M_{a}=\bigcup_{\beta} \bar{P}_{\beta}$. Since $\bar{D}_{M_{\alpha}}$ is not a quotient ring of $D$, we have $\bar{D}_{\hat{M}_{\hat{\alpha}}} \subset D_{P_{\beta}}=\bar{D}_{\bar{P}_{\beta}}$. Thus $\bar{P}_{\beta} \subset M_{\alpha}$ for each $\beta$, and $M_{\alpha}$ is the union of a chain of prime ideals of $\bar{D}$ which are properly contained in $M_{\alpha}$. Since $\bar{D}$ is a 
Prüfer domain, it follows that $M_{\alpha}$ is unbranched.

(b) $\rightarrow$ (c): It is clear that there are no domains properly between $D$ and $\bar{D}$. Corollary 2.2 shows that $\bar{D}$ has at most two maximal ideals. If $\bar{D}$ has two maximal ideals then by Proposition 2.5, $\bar{D}$ is a Prüfer domain. If $\bar{D}$ is quasi-local then $M \bar{D}$ has radical $\bar{M}$, the maximal ideal of $\bar{D}$. By Lemma $2.3, M \bar{D}=M$ and by hypothesis $\bar{M}$ is unbranched; hence $M=\bar{M}$. It follows in this case from Proposition 2.6 that $\bar{D}$ is Prüfer. Since $M$ is the conductor of $D$ in $\bar{D}$, Lemma 3.2 shows that $M$ is unbranched as an ideal of $D$.

(c) $\rightarrow$ (a): Theorem 2.4 shows that $\bar{D}$ is the unique minimal overring of $D$. Let $D_{1}$ be an overring of $D$. If $D=D_{1}$, then $D_{1}=D_{M}$. If $D \subset D_{1}$, then $\bar{D} \subseteq D_{1}$. Since $\bar{D}$ is Prüfer, $\bar{D}$ has the $Q Q R$-property, and there is a set $\left\{\bar{P}_{\beta}\right\}$ of prime ideals of $\bar{D}$ such that $D_{1}=\bigcap_{\beta} \bar{D}_{\bar{P}_{\beta}}$. If a maximal ideal $M_{\infty} \in\left\{\bar{P}_{\beta}\right\}$, then by Lemma 3.1, $M_{\alpha}$ is unbranched and there is a set $\left\{\bar{P}_{\gamma}\right\}$ of non-maximal prime ideals of $\bar{D}$ such that $\bar{D}_{M_{\alpha}}=\bigcap_{\gamma} \bar{D}_{\bar{P}_{\gamma}}$. Therefore $D_{1}=\bigcap_{\lambda} \bar{D}_{\bar{P}_{\lambda}}$ where each $\bar{P}_{\lambda}$ is a non-maximal prime of $\bar{D}$. Hence to show that $D$ has the $Q Q R$-property it will suffice to show that if $\bar{P}$ is a non-maximal prime of $\bar{D}$ then $\bar{D}_{\bar{P}}=D_{P}$ where $\bar{P} \cap D=P$. Since $\bar{D}$ is integral over $D, P$ is a non-maximal prime of $D$ and $\bar{D} \subseteq D_{P}$. Thus $P D_{P} \cap \bar{D}=N$ is a prime ideal of $\bar{D}$ lying over $P$ and $\bar{D}_{N}=$ $D_{P} \subseteq \bar{D}_{\bar{P}}$. Hence $\bar{P} \subseteq N$. It follows that $N=\bar{P}$ and $\bar{D}_{\bar{P}}=D_{P}$. We conclude that $D$ has the $Q Q R$-property.

3.4 COROLLARY. If $J$ is an integral domain with the $Q Q R$ property and if each maximal ideal of $J$ is branched then $J$ is a Prüfer domain.

Proof. If $N$ is a maximal ideal of $J$, then $J_{N}$ has the $Q Q R$ property and $N J_{N}$ is branched. Hence by Theorem 3.3, $J_{N}$ is a valuation ring.

3.5 REMARK. Our results in Section 3 show that in the statement of Theorem 2.7 we can actually say that $M$ is the Jacobson radical of $\bar{D}$. 
4. Examples. We now consider some examples of domains with the $Q Q R$-property which are not Prüfer domains. The examples indicate in some cases negative answers to possible generalizations of results previously obtained.

We denote by $G$ the countable weak direct sum of the additive group of integers, ordered lexicographically. We let $k$ be a field and $k_{0}$ be a subfield over which $k$ is algebraic. As in [7, p. 248] we consider $x_{1}, x_{2}, \cdots, x_{n}, \cdots$ elements of an extension field of $k$ which are algebraically independent over $k$. We define a valuation $v$ on $K=k\left(x_{1}, x_{2}, \cdots\right)$ as follows. For any nonzero element $a$ of $k$ and any nonnegative integers $r_{1}, r_{2}, \cdots, r_{n}$ we define $v\left(a x_{1}^{r_{1}} x_{2}^{r_{2} \cdots} x_{n}^{r_{n}}\right)$ $=\left(r_{1}, r_{2}, \cdots, r_{n}, 0, \cdots\right) \in G ; v(f(x))=$ minimum value of the nonzero monomials occuring in $f(x)$, for any $f(x) \in k\left[x_{1}, x_{2}, \cdots\right]$; and $v(\xi)=v(f)-v(g)$ for any $\xi=f / g \in k\left(x_{1}, x_{2}, \cdots\right)$. Let $V$ be the valuation ring associated with $v$ and let $M_{1}$ be the maximal ideal of $V$. We observe that $V=k+M_{1}$ and that $M_{1}$ is an unbranched ideal of $V$. Consider the domain $D=k_{0}+M_{1} . \quad D$ is a quasi-local domain with maximal ideal $M_{1}$ and integral closure $\bar{D}=V$. Lemma 3.2 shows that $M_{1}$ is unbranched as an ideal of $D$. There is a one-to-one correspondence between domains between $D$ and $\bar{D}$ and fields between $k_{0}$ and $k$.

4.1 EXAMPLE. In the above construction we take $k$ and $k_{0}$ to be fields such that $k_{0} \subset k$ and such that there are no fields properly between $k_{0}$ and $k$. Theorem 3.3 then proves that the domain $D=k_{0}+M_{1}$ has the $Q Q R$-property.

In [2, p. 200], Davis raised what is, in our terminology, this question: Must a domain $D$ with the $Q Q R$-property be Prüfer? Davis showed that the answer is affirmative if each prime ideal of $D$ has finite rank-a result generalized by our Theorem 1.4. And Example 4.1 shows that the answer to Davis' question is negative.

4.2 EXAMPLE. In our construction preceding Example 4.1 we choose for $k_{0}$ and $k$ fields such that there exist proper intermediate fields. Then Theorem 1.10 shows that $D=k_{0}+M_{1}$ does not have the $Q Q R$-property. But $D$ does have the property that 
each valuation ring containing $D$ which is an overring of $D$ is an intersection of quotient rings of $D$. To prove this statement we observe that since $\bar{D}=k+M_{1}$ has unbranched maximal ideal, $\bar{D}$ is an intersection of valuation rings which properly contain $\bar{D}$. Hence it suffices to show that each valuation ring $V$ such that $\bar{D} \subset V \subset K$, is a quotient ring of $D$. Any such $V$ is of the form $(\bar{D})_{P}$ for some prime $P$ of $\bar{D}, P \subset M_{1}$. Then if $m \in M_{1}-P$ and $x \in k, x m \in M_{1}-P$, implying that $x=x m / m \in D_{P}$. It follows that $k \subseteq D_{P}$, and hence that $k+M_{1}=\bar{D} \subseteq D_{P}$. Therefore $D_{P}=\bar{D}_{\left(P D_{P} \cap \bar{D}\right)}$ $=\bar{D}_{P}=V$.

Example 4.2 shows that the condition we considered in Theorems 1.4-1.6 is weaker than the $Q Q R$-property. In Example 4.3 we construct a quasi-local domain $D$ with the $Q Q R$-property such that the integral closure of $D$ is not a valuation ring. This example also shows that the unique minimal overring of a quasilocal domain may not be quasi-local.

4. $3 \mathrm{EXAMPLE}^{4)}$. Let $k$ denote a prime field and let $x_{1}, x_{2}$, $\cdots, x_{n}, \cdots$ be elements of an extension field which are algebraically independent over $k$. We define a valuation $v$ on $k\left(x_{1}, x_{2}, \cdots\right)$ having value group $G$ as in Example 4.1. If we let $y_{i}=x_{i}-1$ for each $i$, then $k\left(y_{1}, y_{2}, \cdots\right)=k\left(x_{1}, x_{2}, \cdots\right)$. Define a valuation $w$ on $k\left(y_{1}, y_{2}, \cdots\right)$ by $w\left(f\left(y_{1}, \cdots, y_{n}\right)\right)=v\left(f\left(x_{1}, \cdots, x_{n}\right)\right) ; w(\xi)=w(f)-w(g)$ where $\xi=f / g \in k\left(y_{1}, y_{2}, \cdots\right)$. We denote by $V$ and $W$ the valuation rings associated with the valuations $v$ and $w$ respectively. If $M_{1}$ and $M_{2}$ are the maximal ideals of $V$ and $W$, we show easily that $V=k+M_{1}, W=k+M_{2}$, and that $M_{1}$ and $M_{2}$ are unbranched. Let $D=k+M$, where $M=M_{1} \cap M_{2}$, and denote by $\bar{D}$ the integral closure of $D$. We show that $\bar{D}=V \cap W$. It suffices to observe that each element $t$ of $V \cap W$ is integral over $D$. Thus there are elements $a, b$ of $k$ such that $t-a \in M_{1}, t-b \in M_{2}$. And since $t-a \in W$ and $t-b \in V$, it follows that $s=(t-a)(t-b) \in M_{1} \cap M_{2}$. Therefore, $t^{2}-(a+b) t+(a b-s)=0$, and $t$ is integral over $D$. It follows that $\bar{D}=W \cap V$. Since $V \neq W, \bar{D}$ is a Prüfer domain with two maximal

4) Constructions like that of Example 4.3 occur in [5] and [10]. In particular, our proof that $\bar{D}=V \cap W$ comes from [10]. 
ideals, $M_{1} \cap \bar{D}$ and $M_{2} \cap \bar{D}[12$, p.56]. We observe that $D=k+M$ is quasi-local with maximal ideal $M$, so that $D \subset \bar{D}$. Because $M=M_{1} \cap M_{2}, M$ is an ideal of $\bar{D}$ and is the conductor of $D$ in $\bar{D}$. We now show that there are no domains properly between $D$ and $\bar{D}$. If $\bar{M}_{i}=M_{i} \cap \bar{D}$ then $\bar{D}_{\bar{M}_{1}}=V$ and $\bar{D}_{\bar{M}_{2}}=W$. Also $\bar{D} / M=\bar{D} / \bar{M}_{1} \cap \bar{M}_{2}$ $\cong \bar{D} / \bar{M}_{1} \oplus \bar{D} / \bar{M}_{2}$ and $\bar{D} / \bar{M}_{1} \cong k$. The inclusion map of $D$ into $\bar{D}$ induces a map of $D / M \cong k$ into $\bar{D} / M \cong k \oplus k$ which we will denote by $\tau$. We have $\tau(1)=1 \oplus 1$ so that $\tau(a)=a \oplus a$ for each $a \in k$. It is easy to see that $k \oplus k$ is a simple ring extension of $\tau(k)$ by any element of $(k \oplus k)-\tau(k)$. Hence for any $d \in \bar{D}-D$, we have $D[d] / M=\bar{D} / M$. Since $M \subset D$, it follows that $D[d]=\bar{D}$ and there are no rings properly between $D$ and $\bar{D}$. Because $\bar{D}$ is a Prüfer domain, Theorem 2.4 implies that $\bar{D}$ is a unique minimal overring of $D$. Also the valuation rings $V$ and $W$ are the intersection of valuation rings properly containing them. Since $\bar{D}$ is a Prüfer domain, it follows that the maximal ideals of $\bar{D}$ are unbranched. Theorem 3.3 then shows that $D$ has the $Q Q R$-property.

We next give an example of a domain with the $Q Q R$-property which is neither Prüfer nor quasi-local.

4.4 EXAMPLE. We consider $D=k+M$, the quasi-local domain with the $Q Q R$-property which was constructed in Example 4.3. Let $T$ be a valuation ring on $k\left(x_{1}, x_{2}, \cdots\right)$ of the form $k+M_{3}$, where $M_{3}$ is the maximal ideal of $T$, and such that there are no containment relations between $T, V$, and $W$.

4.5 PROPOSITION. $D^{*}=T \cap D$ is a domain with the $Q Q R$ property which is neither quasi-local nor Prüfer.

Proof. By an argument similar to that given in Example 4.3 we show that any valuation ring containing $D^{*}$ contains either $T, V$, or $W$. Therefore the integral closure of $D^{*}$ is $R=T \cap V \cap W$ and the maximal ideals of $D^{*}$ are the centers of $T, V$ and $W$ on $D^{*}$. Since $D^{*} \subseteq D, M_{1} \cap D^{*}=M_{2} \cap D^{*}=P$. Because there are no containment relations among $T, V$, and $W, R$ is a Prüfer domain with distinct maximal ideals $M_{1} \cap R, M_{2} \cap R$, and $M_{3} \cap R$. Therefore $D^{*} \subset R$ and $D^{*}$ is not Prüfer. Let $N=M_{3} \cap D^{*}$. We show that $D^{*}{ }_{N}=T$ and $D^{*}{ }_{P}=D$. Since $R_{M_{3} \cap R}=T$, if $x \in T$, then $x=a / b$ 
where $a, b \in R$ and $b \notin M_{3} \cap R$. We choose $y \in\left(R \cap M_{1} \cap M_{2}\right)-M_{3}$. Then $x=a y / b y, a y \in T \cap D=D^{*}, b y \in D^{*}$, and $b y \notin D^{*} \cap M_{3}$. Therefore $x \in D^{*}{ }_{N}$ and we conclude that $D^{*}{ }_{N}=T$. Since $D^{*}{ }_{P} \subseteq D$, it is clear that $P \neq N$ and $D^{*}$ is not quasi-local. If $x \in D$, we show there exists $y \in D^{*}-P$ such that $y x \in D^{*}$. If $x \in T$, then $x \in T \cap D=D^{*}$ and we take $y=1$. If $x \notin T$, then $x^{-1} \in M_{3}$. Let $Q$ be the radical of $x^{-1} T, Q$ is a prime ideal of $T$ and $Q \cap D^{*} \nsubseteq$ $M \cap D^{*}$. This follows because $R$ is integral over $D^{*}$ and $Q \cap R$ is a prime ideal of $R$ lying over $Q \cap D^{*}$. Thus by the "going up" theorem [11, p. 749], if $Q \cap D^{*} \subseteq M \cap D^{*}$, then $Q \cap R$ is contained in a prime ideal of $R$ lying over $M \cap D^{*}$. But $M_{1} \cap R$ and $M_{2} \cap R$ are the only prime ideals of $R$ lying over $M \cap D^{*}$ and since $R_{Q \cap R}=T_{Q}, R_{M_{1} \cap R}=V$, and $R_{M_{2} \cap R}=W$, if either $M_{1} \cap R$ or $M_{2} \cap R$ contains $Q \cap R$, then either $V$ or $W$ is contained in $T_{Q}$. But $x \in V \cap W$ and $x \notin T_{Q}$ since $x^{-1} \in Q$. Therefore $Q \cap D^{*}$ is not contained in $M \cap D^{*}$. We choose $a \in\left(Q \cap D^{*}\right)-M$. There is a positive integer $n$ such that $a^{n} \in x^{-1} T$. If $y=a^{n}$, then $y \in D^{*}-M$ and $y x=$ $a^{n} x \in T$. Therefore $y x \in D^{*}=T \cap D$. We conclude that $D^{*}{ }_{M \cap D^{*}}=D$. It follows from Theorem 1.9 that $D^{*}$ has the $Q Q R$-property.

4.6 REMARK. If $D_{1}, D_{2}, \cdots, D_{n}$ are quasi-local domains with the $Q Q R$-property such that each $D_{i}$ has quotient field $K$, then $D=\cap_{i=1}^{n} D_{i}$ also has $K$ as its quotient field. For if $M_{i}$ is the maximal ideal of $D_{i}$, then $M=\cap_{i=1}^{n} M_{i}$ is the Jacobson radical of $\cap_{i=1}^{n}\left(V_{i} \cap W_{i}\right)$ where $V_{i}$ and $W_{i}$ are valuation rings of $K$ such that $V_{i} \cap W_{i}$ is the integral closure of $D_{i}$ (we may have $V_{i}=W_{i}$ for some $i$ ). Since $M$ is an ideal of $D, D$ and $\cap_{i=1}^{n}\left(V_{i} \cap W_{i}\right)$ have the same quotient field. Therefore $D$ has quotient field $K$. The intersection, however, of a finite number of quasi-local domains with the $Q Q R$-property need not have the $Q Q R$-property. In fact our final example is an integral domain $D$ which is an intersection of two quasi-local domains with the $Q Q R$-property each having quotient field $K$ such that the integral closure of $D$ is not Prüfer.

4.7 EXAMPLE. Let $F$ be a field of characteristic zero and let $y$ be transcendental over $F$. We construct as in Example 4.1 a valuation $\operatorname{ring} V=F(y)+M$, where $M$, the maximal ideal of $V$, 
is unbranched. $F(y)$ is an algebraic field extension of dimension 2 over each of the fields $F\left(y^{2}\right)$ and $F\left(y^{2}+y\right)$. Therefore $D_{1}=F\left(y^{2}\right)+M$ and $D_{2}=F\left(y^{2}+y\right)+M$ are quasi-local domains with the $Q Q R$ property. Since the sum of $F(y)$ and $M$, as additive subgroups of $V$, is direct, we have $D=D_{1} \cap D_{2}=\left(F\left(y^{2}\right) \cap F\left(y^{2}+y\right)\right)+M$. But it is not hard to show that $F\left(y^{2}\right) \cap F\left(y^{2}+y\right)=F[9, \mathrm{p} .31]$. Therefore $D=F+M$ is an integrally closed quasi-local domain which is not Prüfer.

\section{REFERENCES}

[1] N. Bourbaki, Elements de Mathematique, Algebre Commutative, Chapitre 5-6, Paris, Hermann (1964).

[2] E. D. Davis, Overrings of commutative rings. II, Trans. Amer. Math. Soc. 110 (1964), 196-212.

[3] R. W. Gilmer, A class of domains in which primary ideals are valuation ideals, Math. Ann. 161 (1965), 247-254.

[4] — On overrings of Prüfer domains, J. of Algebra, 4 (1966), 331340.

[5] R. W. Gilmer and W. J. Heinzer, Primary ideals and valuation ideals II, to appear in Trans. Amer. Math. Soc.

[6] R. W. Gilmer, and J. E. Ohm, Integral domains with quotient overrings, Math. Ann. 53 (1964), 97-103.

[7] - Primary ideals and valuation ideals, Trans. Amer. Math. Soc., 117 (1965), 237-250.

[8] O. Goldman, On a special class of Dedekind domains, Topology, 3 (1964), 113-118.

[9] N. Jacobson, Lectures in Abstract Algebra, Vol. III, Princeton, D. Van Nostrand Company (1964).

[10] T. Kikuchi, Some remarks on S-domains, J. Math. Kyoto Univ. 6 (1966), $49-60$.

[11] W. Krull, Beiträge zur Arithmetik kommutativer Integritätsbereiche, III, Math. Z. 42 (1937), 745-766.

[12] M. Nagata, On the theory of Henselian rings, Nagoya Math. J. 7 (1954), $45-57$.

[13] — Local Rings, New York, Interscience, 1962.

[14] J. Ohm, Primary ideals in Prüfer domains, Canad. J. Math. 18 (1966), 10241030.

[15] R. L. Pendleton, A characterization of Q-domains, Bull. Amer. Math. Soc., 72 (1966), 499-500.

[16] F. Richman, Generalized quotient rings, Proc. Amer. Math. Soc. 16 (1965), 794-798.

[17] O. Zariski and P. Samuel, Commutative Algebra, Vol. I, Princeton, D. Van Nostrand Company (1958).

[18] - Commutative Algebra, Vol. II, Princeton, D. Van Nostrand Company (1960). 
Florida State UNIVERSity and

Louisiana State UnIVERSity 